\title{
Comparison of induction of B45 Helicobacter pylori prophage by acid and UV radiation
}

\author{
A.P. Alves de Matos*, P. Lehours**, A. Timóteo******** M. Roxo-Rosa************ and F.F .Vale*** \\ *Anatomia Patológica, Centro Hospitalar de Lisboa Central - HCC, Rua da Beneficência 8, 1069-166 Lisboa, \\ Portugal; Centro de Estudos do Ambiente e do Mar (CESAM/FCUL) - Faculdade de Ciências da Universidade \\ de Lisboa and Centro de Investigação Interdisciplinar Egas Moniz (CiiEM), Quinta da Granja , Monte de \\ Caparica, 2829-511 Caparica, Portugal. \\ **Université Bordeaux Segalen, INSERM U853, 146 Rue Leo Saignat, 33076 Bordeaux, France. \\ ***Faculdade de Engenharia, Universidade Católica Portuguesa, Estrada Octávio Pato, 2635-631 Rio de Mouro, \\ Portugal. \\ **** Faculdade de Ciências, Universidade de Lisboa, Campo Grande, 1749-016 Lisboa, Portugal. \\ *****Departamento de Doenças Infeciosas, Instituto Nacional de Saúde Dr. Ricardo Jorge (INSARJ), Av. Padre \\ Cruz, 1649-016 Lisboa, Portugal \\ ******BioFIG, Center for Biodiversity, Functional \& Integrative Genomics, Faculdade de Ciências, \\ Universidade de Lisboa, Campo Grande, 1749-016 Lisboa, Portugal
}

Helicobacter pylori is a Gram-negative microorganism that grows on microaerophilic conditions and has only one known natural reservoir: the gastric mucosa. The infection by H. pylori is very common worldwide and this bacterium is associated with the development of gastritis, peptic ulcer gastric cancer or gastric Mucosa Associated Lymphoid Tissue (MALT) lymphoma. Although its natural habitat is the acidic gastric mucosa, $H$. pylori is considered to be a neutralophile. The bacterium survives brief exposure to $\mathrm{pHs}$ of $<4$, but growth occurs only at the relatively narrow $\mathrm{pH}$ range of 5.5 to 8.0 , with optimal growth at neutral $\mathrm{pH}[1,2]$. Recently we have identified a prophage sequence (prophage phiHP33) in the strain B45, isolated from a patient diagnosed with gastric MALT lymphoma. This prophage revealed to be very difficult to induce. In fact, only few phage particles were observed on electron microscopy micrographs after exposure to UV radiation [2].

In the present work we have compared the exposure to UV and to acidic environment in the induction of the prophage into a lytic cycle. We have tested two strains, the strain B45 carrying the prophage phiHP33 and a clinical strain 1152, isolated from a patient with peptic ulcer, that was revealed to be negative for the presence of integrase gene (a prophage gene essential for genome integration of prophage) by PCR, as negative control. Since the H. pylori reservoir is the human stomach the exposition to acid is very common, and with this experiment we intended to test if acid can trigger a phage lytic cycle.

The induction using UV radiation has been previously described [2,4]. For acid induction we have used a protocol adapted from Karita and Blaser [3]. A 48 hours culture of $H$. pylori was grown in Brucella broth (Oxoid) supplemented with $10 \%$ of fetal bovine serum (Gibco) and 1\% of Polivitex (BioMérieaux) in microaerophilic conditions at $37^{\circ} \mathrm{C}$. The liquid culture was centrifuged and the cell pellet ressuspended in citratephosphate buffer $\mathrm{pH} 6$ and incubated 15 minutes, centrifuged again and ressuspended in citrate-phosphate buffer pH 3 and incubated for 30 minutes. After centrifugation the supernatant was recovered and incubated for 3 hours in phage precipitant (33\% polyethylene glycol [PEG], 3M NaCl). After centrifugation at $10000 \mathrm{rpm}$ for 10 minutes at $4^{\circ} \mathrm{C}$ the pellet was ressuspended in phage buffer [2]. These samples were analysed by transmission electron microscopy (TEM) after negative staining with $1 \%$ aqueous uranyl acetate, using a JEOL 100SX electron microscope.

For B45 strain the induction using UV radiation (previously reported in [2]) and acid exposure produced similar results (Figure 1 and Figure 2) showing numerous phage-like particles of about $100 \mathrm{~nm}$ diameter, apparently lacking a tail, after UV or acid exposition, respectively. These particles were not observed in the control strain 1152. Currently we are analysing the samples using molecular biology techniques and fixation embedding followed by ultrathin sectioning for TEM analysis, to detect the presence of phages. 
These preliminary results suggest that acid also appears to induce the H. pylori prophage phiHP33. However, since the number of phage particles observed is small, we can not rule out that the observed particles were released spontaneously. The exposition to the natural acidic environment of the human stomach may induce $H$. pylori prophage into a lytic cycle and to the propagation of phages among different $H$. pylori strains colonizing the same individual. Although highly speculative, transduction may be another form of horizontal gene transfer, which has not been described for this bacterium yet.

\section{References}

1. Kusters J.G. et al., Clinical Microbiology Reviews, 19:449, 2006.

2. Lehours P. et al., MBio, 2: pii: e00239-11, 2011.

3. Karita M. and Blaser M.J., The Journal of Infectious Diseases, 178:213, 1988.

4. Vale F.F. et al., Microscopy and Microanalysis, 14(S3):150, 2008.
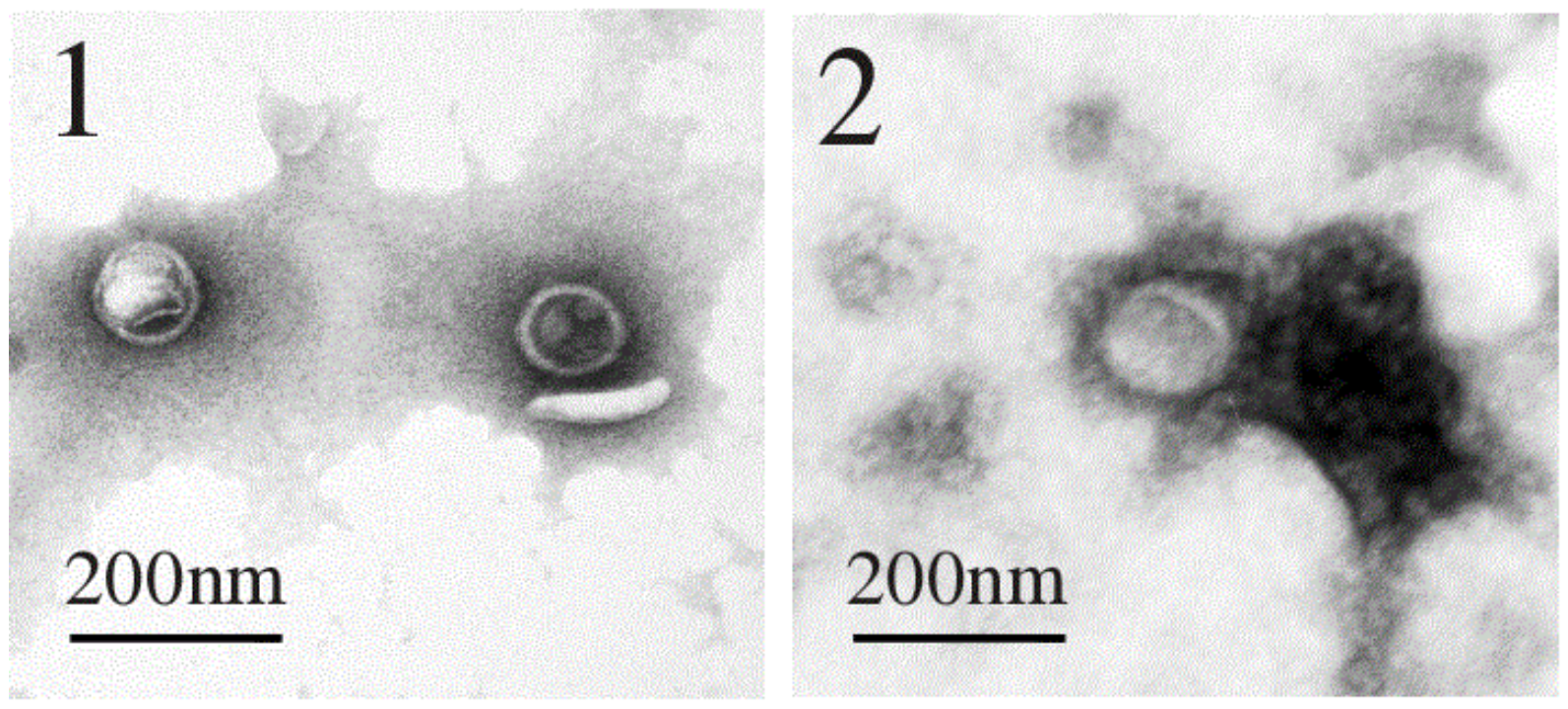

Figure $1 \mathrm{H}$. pylori phage phiHP33 induced by acidic exposure to $\mathrm{pH} 3$ observed by TEM after negative staining.

Figure 2 H. pylori phage phiHP33 induced by UV exposition observed by TEM after negative staining.

Financial support received from the Portuguese Science and Technology foundation under the contract PTDC/EBB-EBI/119860/2010. 